\title{
Day-night and seasonal variation of human gene expression across tissues
}

Valentin Wucher ${ }^{1,4}$, Reza Sodaei ${ }^{1,4}$, Raziel Amador ${ }^{1}$, Manuel Irimia ${ }^{1,2,3,5}$, Roderic Guigó ${ }^{1,2,5}$

1 - Centre for Genomic Regulation, Barcelona Institute of Science and Technology, Barcelona, Spain.

2 - Universitat Pompeu Fabra, Barcelona, Spain.

3 - ICREA, Barcelona, Spain.

4 - Co-first authors.

5 - Corresponding authors.

\section{Manuel Irimia}

Centre for Genomic Regulation

Dr. Aiguader, 88, 08003 Barcelona, Spain

e-mail:mirimia@gmail.com

Phone: +34933160212, Fax: +34933160099

\section{Roderic Guigó}

Centre for Genomic Regulation

Dr. Aiguader, 88, 08003 Barcelona, Spain

e-mail: roderic.guigo@crg.eu

Phone: +34933160168, Fax: +34933160099 


\begin{abstract}
Circadian and circannual cycles trigger physiological changes whose reflection on human transcriptomes remains largely uncharted. We used the time and season of death of 932 individuals from GTEx to jointly investigate transcriptomic changes associated with those cycles across multiple tissues. For most tissues, we found little overlap between genes changing expression during day-night and among seasons. Although all tissues remodeled their transcriptomes, brain and gonadal tissues exhibited the highest seasonality, whereas those in the thoracic cavity showed stronger day-night regulation. Core clock genes displayed marked day-night differences across multiple tissues, which were largely conserved in baboon and mouse, but adapted to their nocturnal or diurnal habits. Seasonal variation of expression affected multiple pathways and were enriched among genes associated with SARS-CoV-2 infection. Furthermore, they unveiled cytoarchitectural changes in brain subregions. Altogether, our results provide the first combined atlas of how transcriptomes from human tissues adapt to major cycling environmental conditions.
\end{abstract}

\title{
Introduction
}

The yearly and daily movement of the earth around the sun and around itself has created a continuously changing environment since the origin of life to which all organisms across the phylogenetic spectrum have adapted. In mammals, in particular, behavioral adaptations to daily rhythm include the regulation of sleep and feeding cycles. Recent studies have investigated how the physiological responses to the daily cycle are reflected at the transcriptomic level. These studies have reported large-scale circadian gene expression oscillations in mice (1), baboons (2), and humans (3). Unlike the circadian process, circannual rhythm in mammals has been less studied. Animals exhibit a range of behavioral and physiological adaptations in different seasons, such as hibernation and alterations of the coat color in polar animals $(4,5)$. One way in which mammals regulate their seasonal reproductive behavior, growth, food intake, and migratory behavior is via the brain-gonadal and other hormonal axes (6-8). In humans, numerous pathologies present a strong seasonal pattern, which is particularly prominent for many infectious diseases, but also observed in complex cardiovascular and psychiatric disorders (9-17). However, despite its relevance for human 
physiology and disease, there are, to our knowledge, no genome-wide studies of the transcriptional impact of these adaptations to seasonal variation on human tissues.

Here, we leverage on the deep transcriptome data across human tissues produced by the GTEx consortium (18) to investigate the transcriptional impact of circadian and circannual rhythms in an unprecedented number of tissues. GTEx transcriptional measurements are taken exclusively at the donor's death; therefore, there is a single time-point measure per individual. However, we show that, when aggregated over many individuals, these transcriptional snapshots randomly distributed along time create temporal trajectories that recapitulate circadian and circannual transcriptional variation.

\section{Results}

\section{Profiling day-night and seasonal transcriptomic variation from GTEx data}

We used 16,151 RNA-seq samples of 932 post-mortem human donors from 46 tissues from the Genotype-Tissue Expression Project (GTEx v8) (18) to assess how human transcriptomic programs change with day-night and seasonal cycles (Fig. 1, Methods). GTEx metadata only includes the time of the day and the season of death, but not the actual day, the week or even the month of death. This has forced us to discretize circadian and circannual variation. The time of death was used to classify individuals as either dead during the day [8:00-17:00) (351 individuals) or dead during the night [21:00-5:00) (315 individuals) (Fig. 1A). Individuals for whom the time of death fell outside these two intervals were discarded from the day-night analysis due to the variation of sunset and sunrise hours throughout the year and among locations. Thus, strictly speaking, we did not investigate circadian gene expression patterns, but day-night patterns. For the seasonal analysis, we grouped individuals based on their death season (spring: 190 individuals; summer: 221 individuals; fall: 282 individuals; and winter: 239 individuals; Fig. 1B). Differential gene expression analysis was carried out using voom/limma (19) controlling for the effects of sex, body mass index, age, and post-mortem interval, and, for the seasonal analysis, we also included the classification of the time of death to control for the influence of day-night variations, including all individuals (see Methods).

\section{Tissue-dependent day-night variation in gene expression}


From the 18,022 protein-coding genes expressed in at least one tissue (median TPM $\geq 1$ ), $14,002(77.7 \%)$ were differentially expressed between day and night in at least one of the 46 tested tissues (Fig. 2A). This number of day-night genes was in line with a previous study in baboon, showing that $81.7 \%$ of protein-coding genes had circadian patterns in at least one of the 64 studied tissues (2). At the individual tissue level, 8.4\% protein-coding genes changed on average during day-night. The tissue with the largest number of differentially expressed genes was lung, with 4,062 genes (29\% of all genes expressed in this tissue, Fig. 2A). Other tissues with a proportionally large number of day-night genes were esophagus muscularis (25.1\%) and heart left ventricle (24.2\%). On the other hand, the tissue with fewer differentially expressed day-night genes was salivary gland, with only 76 genes $(0.6 \%$ of all genes expressed in this tissue, Fig. 2A). Other tissues with proportionally low number of daynight genes were the small intestine (1.4\%) and testis $(1.9 \%)$. In general, brain regions also showed a small number of day-night changes (ranging from $1.2 \%$ to $7.8 \%$; Fig. 2A). Cerebellum, in contrast, was among the tissues with the largest percentage of day-night genes, $11.6 \%$ ), consistent with sleep stage-dependent activity reported for this organ (20). Interestingly, some tissues showed a bias in the number of genes overexpressed at night (nocturnal genes) versus day (diurnal genes)(Fig. 2A and Table S1). For instance, many neural tissues had preferential diurnal expression: cerebellum and pituitary, in particular, had twice as many diurnal than nocturnal genes. On the other hand, testis exhibited a very strong nocturnal preference.

\section{Recurrent day-night variation in gene expression across tissues}

We next computed the number of tissues in which a given gene showed a day-night pattern (Fig. 2B). We found that, on average, genes exhibited a day-night pattern in 3.6 tissues out of 40 tissues in which genes had detectable expression (median TPM $\geq 1$ ), indicating a rather tissue-specific response to day-night cycles. The gene with a day-night pattern in the largest number of tissues (34) was ARNTL (Fig. 2C), which is well known to have circadian behaviour. The expression at the time of death, aggregated across the GTEx donors, nicely captures the known circadian behaviour of this gene, strongly indicating that, in spite of all the caveats associated with the data collection and available metadata, the GTEx data can be effectively used to investigate gene expression patterns during the day/night cycle. This pattern also indicates that our approach is rather conservative and may underestimate the 
circadian effect size, since, as illustrated in the case of $A R N T L$, the largest difference in gene expression might occur between the twilight zones, which were not considered here.

We focused on the day-night gene expression changes of a set of 16 genes known as core clock genes, whose protein products are involved in generating and regulating the circadian clocks according to several studies (21-23) (Table S2). As expected, with the exception of $R O R B$, core clock genes showed differential day-night expression in multiple tissues (Fig. 2B). Moreover, they usually have effect sizes (difference between day and night expression) that are larger than those of non-core clock day-night genes (Fig. S1). Nine out of the 16 core clock genes had day-night patterns in at least 15 tissues, and, conversely, the eight genes that exhibited significant day-night gene expression differences in at least 20 tissues were all core clock genes. This is in agreement with the hypothesis that one molecular clock program is present in all tissues, but the circadian processes triggered downstream are highly tissuespecific (24). Among the core clock components, the day-night gene expression patterns of $A R N T L$ and NPAS2 were particularly widespread among tissues (in 34 and 30 tissues, respectively; Fig. 2C,D). A recent study described $A R N T L$ as the main regulator of the intertissue timekeeping function in mouse (25), and our results suggest that it may play a similar role in humans. NPAS2 has also been reported as highly circadian in mouse (26) and baboon (2).

In humans, the thyroid had the highest number of changes in the core clock genes between day and night (12 out of 16 genes; Fig. 3A). In contrast, we did not detect any of them as daynight in testis and vagina, consistent with their overall low number of day-night changes (Fig. 2A) and with previous studies in testis in rodents (27). Clock genes showed a largely consistent pattern of day-night expression across tissues (i.e. they were either consistently upor down-regulated at the same time of the day; Fig. 3A). One exception was NR1D2, whose expression was higher diurnally in brain subregions but nocturnally in all other tissues (Fig. 3B and Fig. S2). For the core clock genes that showed day-night differences in both human and baboon in any of the available 20 homologous tissues (Table S3), we found that most orthologs had a similar behavior in both species (57 similar vs. 15 opposite gene-tissue pairs; $P=3.27 \times 10^{-7}$ one-sided binomial test; Fig. 3C), consistent with their shared diurnal regimes. In contrast, core clock genes in mouse (a preferentially nocturnal mammal) largely showed the 
opposite behavior than their human counterparts (15 similar vs. 38 opposite gene-tissue pairs; $P=1.10 \times 10^{-3}$ one-sided binomial test; Fig. $3 \mathrm{C}$ and Table S4).

We found other protein-coding genes exhibiting day-night patterns in a large number of tissues: 260 protein-coding genes showed significant day-night variation in at least 10 tissues, as for TIMELESS (Table S5). These genes were enriched in circadian and melatonin regulation Wikipathways (Fig. 3D), but only 44 have previously been annotated as circadian in the Circadian Gene DataBase in human (28). We observed that these genes were more prone to be either mostly day or mostly night consistently across tissues, compared with genes with day-night changes in fewer than ten tissues (Fig. S3). An example is THRA, a thyroid hormone receptor that peaks at night in 17 tissues, the expression of which was found disrupted in the hypothalamic structures of rats in constant darkness or lighting (29). Similarly, $S M C 3$, a subunit of the cohesin complex, was highly expressed at night in 16 tissues. Down-regulation of the cohesin subunit $S m c 3$ in mouse embryonic fibroblasts led to the down-regulation of the core clock gene $\operatorname{Nrldl}$ (30). Another example of widespread daynight expression variation is the diurnal increase of two nonsense mediated decay (NMD) factors, UPF1 and SMG9, in both human and baboon. Preußner et al. showed the influence of the day-night body-temperature on the splicing regulation (31). Some of the genes present in a large number of tissues (at least 15) have an effect size comparable to that of core clock genes (Fig. S4): the ribosomal protein RPS26 (day in 17 tissues), the nuclear pore complex protein NPIPB5 (day in 16 tissues), and the transcription corepressor TRIM22 (day in 14 tissues, night in one).

One of the signatures of the day-night rhythmicity is the sleep-wake cycle. Thus, we next focused on a set of 254 protein-coding genes expressed in GTEx and that were previously reported to increase the risk of insomnia or to be associated with other sleep traits in humans (32) (Table S6). From this gene set, 210 (82.6\%) exhibited a significant day-night variation in at least one tissue, closely matching the genome-wide behavior across tissues (Fig. S5). However, we found that four of these genes, ZMIZ2, KDM3B, QSOX2, and RASD1, fluctuated in ten or more tissues (Table S6), and were annotated as circadian in Circadian Gene DataBase (28). For instance, ZMIZ2, associated with "ease of waking up in the morning" (32), had a higher expression during the day in 13 tissues (although not in the brain; Fig. S6A), and 
$K D M 3 B$, associated with "sleep duration" (32), had an increase of expression exclusively during the night in 12 tissues (Fig. S6B). The day-night pattern of expression of these genes can contribute to the understanding of the mechanisms by which they may impact sleep traits.

\section{Tissue-dependent seasonal variation in gene expression}

Since the GTEx metadata only reports the season of death, we identified genes that were differentially expressed in a particular season by comparing expression levels in each season against the other three, minimizing the impact of measurements taken around the seasonal boundaries (see Methods). Overall, we found that 92\% $(16,572)$ of all expressed proteincoding genes were differentially expressed in at least one season in at least one tissue (hereafter seasonal genes; Fig. 4A). There were no large differences in the number of seasonal genes across seasons, ranging from 13,963 genes in winter to 14,814 in fall. Per tissue and per season, the average number of seasonal genes were similar among seasons and comparable to day-night patterns $(7.8 \%$ in spring, $8.2 \%$ in summer, $10.4 \%$ in fall, and $7.6 \%$ in winter, compared with $8.4 \%$ day-night genes), but there were many more unique seasonal genes than day-night genes per tissue when all seasons were considered together $(26.3 \%)$. The effect sizes of seasonal genes were also similar to those observed in day-night genes (Fig. S7).

In stark contrast to day-night patterns, the tissue with the highest proportion of genes showing seasonal changes was testis $(50.9 \%$ of all its expressed genes; Fig. 4A), with the highest variation occurring in fall and spring (Fig. S8). Other tissues with large seasonal changes included artery tibial (spring and winter), adipose subcutaneous (summer and fall), breast (spring, summer and winter), skin (spring and summer) and many brain subregions (summer, fall and winter). Most of these tissues did not show a clear bias in the direction of the expression changes (i.e. the number of season-specific up- or down-regulated genes was similar; Fig. S8 and Table S7). However, testis exhibited a massive gene up-regulation in fall and down-regulation in spring, while artery tibial showed a large down-regulation in winter, in contrast with milder up-regulation in the rest of the seasons.

Interestingly, there was little overlap between the day-night and seasonal genes for most tissues (Table S8), as well as between the tissues with larger day-night and seasonal variation in gene expression, as illustrated by the lack of correlation between the numbers of day-night 
and seasonal genes (Fig. 4B). Tissues with larger day-night than seasonal variation included various tissues from the thoracic cavity (e.g. lung and the heart's left ventricle), which may reflect changes in heart rate and breathing patterns between day and night (33). On the other hand, tissues with more seasonal than day-night genes included most brain subregions and gonadal tissues, likely mirroring the involvement of brain-gonadal axis in regulating seasonal physiology and behavior (34) (Fig. 4C).

\section{Recurrent seasonal variation of gene expression across tissues}

On average, genes showed seasonal expression in a number of tissues that is comparable to that of day-night genes (3.3 in spring, 3.6 in summer, 4.3 in fall, and 3.3 winter, compared with 3.6 tissues for day-night genes). Many genes showed consistent seasonal expression in multiple tissues: 1,370 genes had seasonal expression in at least ten tissues (Fig. 5A, Tables S9 and S10). Although these genes showed enrichment for Wikipathway functions that were mainly specific for individual seasons (e.g. muscle hypertrophy in winter), functions related to cytoplasmic ribosomes, methionine metabolism, and oxidative phosphorylation were enriched in spring, summer, and fall (Fig. 5B). Methionine pathways have been described to be involved in innate immune response, digestive functioning, counteracting oxidative stresses and DNA damage, and possibly preventing arterial, neuropsychiatric, and neurodegenerative disorders in mammals $(35,36)$. Consistent with the little overlap between seasonal and daynight genes, there were no shared enriched functions between seasonal and day-night genes.

Eight seasonal genes were highly recurrent (seasonal in at least 20 tissues, or in more than 15 non-brain tissues): five in the fall, two in spring and one in winter (Fig. 5A). One example is GLTSCR1, a component of the SWI/SNF chromatin remodeling complex also known as $B I C R A$, which increased in fall in 23 tissues (e.g. in cerebellar hemisphere, inset of Fig. 5A). Similarly, RTF1, a component of the RNA polymerase II transcription-associated PAF1 complex, decreased in fall in 23 tissues (Fig. S9A). This complex is deeply conserved across eukaryotes, and it has been described to be involved in the regulation of flowering time in plants $(37,38)$ and to be required for induction of heat shock genes in animals $(39,40)$. PAF1c has been proposed to establish an antiviral state to prevent infection by incoming retroviruses: in case of infection by influenza A strain H3N2, PAF1c associates with viral NS1 protein, thereby regulating gene transcription (41). Other examples include $C 4 A$, decreasing in 
spring in 21 tissues (Fig. S9B), which localizes to the histocompatibility complex, and KRT1, which decreases in 24 tissues in winter (Fig. S9C), and encodes a keratin gene that is a downstream effector of the corticotropin hormone release pathway (42). Krt1 mutations in mouse have been associated with various phenotypic defects, ranging from abnormal circulating interleukin (43) to aberrant pigmentation of the epidermis (44). Seasonal pigment variation is well known in mammals from the northern hemisphere (45), which might suggest a conserved role of the corticotropin release pathway in pigment seasonal variation.

We next focused on a set of 192 genes (1.1\% of all expressed genes) that exhibited the strongest quantitative seasonal expression differences (at least a two-fold change in expression in one tissue/season pair). These were usually highly tissue specific, since only seven of these genes belonged to the set of 1,370 genes with recurrent seasonal patterns. These genes were enriched for functions related to skin regeneration and immunity (Fig. 5C). Moreover, they exhibited significant overlap with gene sets related to COVID-19, including genes whose expression changes upon SARS-CoV-2 infection and genes predicted to be functionally related to $A C E 2$ (Fig. 5D), although $A C E 2$ itself did not show a strong seasonal pattern. In particular, 25 out of the top 200 genes among the latter predictions (12.5\%) were strongly seasonal, with 20 of them being up-regulated in the intestine specifically in the fall.

\section{Seasonal variation of gene expression of hormone genes}

Hormones $(46,47)$ have been described to broadly regulate the body's seasonal physiology (6-8). Thus, to explore whether genes that encode peptide hormones undergo particularly strong seasonal changes, we used a list of 62 genes with hormone-encoding capability based on Mirabeau et al. (48) (Table S11). Overall, we found 39 hormone genes to be seasonal in at least one tissue (Fig. S10), and eight were seasonal in at least ten tissue/season pairs (Fig. 5E). Among these, $P O M C$, a well-known photoperiodic hormone, was seasonal in 26 tissue/season pairs, mainly in summer. POMC expression has been shown to be dependent on longer-term photoperiod in the Siberian hamsters (49). Other seasonal hormones also have well known roles, mainly in the cardiovascular system and growth: UCN, a corticotropin for stress response and appetite regulation $(50,51), N P F F$, which controls heart rate and blood pressure (52), and $A D M$, which is important for vasodilation (53). GNRH1, the gonadotropin-releasing hormone 1, which influences seasonal changes in other mammals (54), is seasonal in two 
artery tissues: the aorta and the tibial one. Mutations in GNRH1 have been shown to be related to ischemic heart disease, which shows a seasonal pattern $(55,56)$. Interestingly, leptin (LEP), a hormone involved in seasonal food-seeking behaviour, thermoregulation (57), and obesity $(58,59)$, was altered only in winter in three tissues: adipose visceral, nerve, and blood (Fig. S10). As expected, the tissue in which the largest number of seasonal hormone genes were robustly expressed (median TPM $\geq 5$ ) was the hypothalamus (19 genes, Fig. S11A,B). Pituitary was also among the tissues with a large number of seasonal hormones (10), together with testis (13) and frontal cortex (12).

\section{Seasonal changes in brain cytoarchitecture}

Various studies have shown seasonal histological variation in different brain regions from several mammals, including anterior cingulate cortex in shrews (60), dendritic spines in amygdala in response to short days (i.e. in fall) in white-footed mice (61) and the volume of suprachiasmatic nucleus in humans (62). To investigate potential seasonal changes in human brain's cytoarchitecture, we used gene expression profiles of cell-type specific markers for a variety of brain cell types (63): astrocytes, neurons, oligodendrocytes, microglia, and endothelial cells (Table S12). We found that astrocyte markers increased their expression in fall and decreased in summer (Fig. 6). In particular, we observed increased expression of astrocyte markers in hypothalamus, frontal cortex, and, to a lesser extent, anterior cingulate cortex in the fall, and a decrease in the cerebellum and frontal cortex in the summer. Oligodendrocyte markers, in contrast, tended to decrease expression in the fall, in particular in basal ganglia and anterior cingulate cortex (Fig. 6). In winter, all oligodendrocyte markers increased their expression in nucleus accumbens. Moreover, we observed an increase in the expression of neuronal markers in winter in the hypothalamus, the spinal cord, and the anterior cingulate cortex (Fig. 6). These results are thus consistent with previous histological studies in humans and other mammals (60-62) that showed that the relative volume or cytoarchitecture of astrocytes, oligodendrocytes and neurons change with the season in a subregion-specific manner.

\section{Discussion}

By leveraging the rich transcriptome data produced by the GTEx project, we have investigated the impact of the circadian and circannual cycles in the human transcriptome across multiple 
tissues. Even though GTEx captures a single snapshot of the tissue transcriptomes of the donors at the time of death, since this time is approximately uniformly distributed along time, the aggregation of the data snapshots across individuals produces trajectories that allowed us to investigate temporal variations in gene expression. Moreover, although there is an impact of the death of the individual in the transcriptome, which is tissue-specific, this can be properly controlled for (64). The GTEx medatada, however, by making accessible only the time of the day and the season of death of the donors makes this investigation challenging. We have addressed this limitation by discretizing the continuous circadian variation into day vs. night, and the circannual variation into seasons. In addition, because the time of the day without knowledge of the actual day of the year and the geographical location in which death occurred does not fully inform whether death occurred under daylight, we excluded the data from donors that died within time intervals when the presence of daylight could not be confidently determined (twilight zone). Therefore, we do not refer to the variations reported here as circadian and circannual, but as day-night and seasonal, respectively. Despite these caveats, our approach was able to properly capture at least part of the real circadian and circannual transcriptional variation, since we have been able to recapitulate previous findings regarding day-night variation.

We found that the effect of day-night variation in gene expression was comparable to that of the seasonal cycle, but affecting very different genes and tissues. Day-night variation in gene expression was more prominent in liver, lung, heart, and upper digestive tract, reflecting the involvement of the organs of the thoracic cavity in circadian processes (65), while seasonal variation had the strongest effect in brain subregions and testis, mirroring the role of the braingonadal hormonal axis in regulating the physiological responses to seasonal variation (66). Moreover, we showed that the effect of day-night and/or seasonal variation for most genes was highly tissue-specific. However, core subsets of genes showed day-night and seasonal expression behaviours across multiple tissues. In the case of the day-night genes, this is in agreement with the hypothesis that one molecular clock program is present in all tissues, but the circadian processes triggered downstream are highly tissue-specific (24). The day-night core subset included most known core circadian clock genes. The direction of the change in gene expression for the core clock genes was largely consistent among tissues, but we found some cases in which changes in gene expression occurred unexpectedly in opposite directions in different tissues. This is, for instance, the case of NR1D2, in which the day-night changes in 
gene expression occurred in the opposite direction in the brain than in tissues from the rest of the body. This suggests that the cellular environment could modulate the interpretation of the core clock signals, and produce different (or even opposite) outputs in different tissues in response to the same environmental cues.

Among all core clock genes, $A R N T L$ and $N P A S 2$ had a differential day-night transcriptional behaviour across the largest number of tissues. In the circadian negative feed-back loop, $A R N T L$ forms an heterodimer with either NPAS2 or its paralog CLOCK, positively regulating the circadian pattern (24). Interestingly, we found that NPAS2 shows day-night gene expression differences in a much larger number of tissues than CLOCK (30 vs. 12 tissues), suggesting a more relevant role for NPAS2 in the circadian modulation across tissues in human. Similarly, NPAS2 was detected as cycling in 23 baboon tissues compared to eight tissues for CLOCK (2). In contrast, mouse Clock and Npas2 cycled in a similar number of tissues (eight and seven out of 12 tested tissues, respectively) (26). Whether this difference is a lineage-specific divergence between primates and rodents, or it may be related to distinct diurnal-nocturnal habits (diurnal for human and baboon, nocturnal for mouse) will require investigation in other mammalian groups. In the latter scenario, however, the differential use of NPAS2 and CLOCK could contribute to establishing the opposite circadian patterns across tissues that we observed between primates and mouse.

At the seasonal level, our results highlight the importance of the brain-gonadal hormonal axis. Many physiological and behavioral changes across seasons such as breeding, mating, molting, foraging, and hibernation $(6,47,67,68)$ are known to be regulated by the endocrine system in mammals and birds, especially by the brain-thyroid and brain-gonadal axes, and can elicit different reactions depending on the receiving tissues. This is reflected in the tissue-specific seasonal variation in gene expression that we have undercovered, which affects prominently testis, breast and many brain regions. When investigating specifically the expression patterns of genes involved in functions related to the hormonal axis, we found these to be predominantly seasonal in core tissues from the endocrine system, such as hypothalamus and pituitary. Among these genes, NCOR1, a nuclear hormone receptor co-repressor that mediates transcriptional repression of thyroid and androgen hormone together with retinoic acid receptors $(69,70)$, was lowly expressed in 19 tissues during fall and highly expressed in 10 
tissues in summer. This observation is in line with the potential role that hormonal receptors regulation can play in adaptation to seasonal variation (47).

Infectious diseases are well-known to have a seasonal pattern, as illustrated, for instance, by the current COVID-19 pandemics. We have found a significant number of genes in various COVID-19 related pathways and associated gene sets to be strongly seasonal in a tissue specific manner. In particular, these included genes that change expression in response to SARS-CoV-2 infection and genes predicted to be functionally related to ACE2, an endogenous membrane protein that mediates SARS-CoV-2 infection. The strong seasonality of these genes could shed light on the molecular mechanisms underlying the seasonality of the virulence of the SARS-CoV-2 infection.

Finally, by evaluating cell type-specific markers, our results suggest a substantial seasonal remodelling of the cytoarchitecture of certain human brain areas. We found a general increase of astrocytes in fall and winter and a decrease in summer across many subregions. These changes in the expression of cell type markers, similar to the volumetric changes described in other mammals, were subregion-specific. Interestingly, we found a reduction of neuronal markers in the anterior cingulate cortex in fall, a subregion whose neuronal soma and dendrite size shrank in the cold season in shrews (60). Whether or not these and other putative cytoarchitectural changes are conserved in other mammals, which are their functional implications, and how they might contribute to the seasonal patterns of some psychiatric and brain diseases $(14,17,61,71,72)$ need to be further investigated.

In summary, our work expands our understanding of the transcriptional impact of the physiological changes associated with the circadian cycle and, for the first time across multiple human tissues, of the circannual cycle. Moreover, these results constitute a large resource for the community to further investigate the impact of day-night and seasonal variation in the human transcriptome.

\section{Methods}

\section{RNA-seq datasets}


The RNA-seq data was generated, mapped and quantified by the GTEx consortium (GTEx v8) (18). Tissues with less than 100 donors were discarded from the analyses (Kidney - Medulla, Kidney - Cortex, Cervix - Ectocervix, Fallopian Tube, Cervix - Endocervix, and Bladder) as well as two cell lines (Cells - EBV-transformed lymphocytes and Cells - Cultured fibroblasts). For the Whole Blood tissue, all pre-mortem samples were discarded from the analysis for homogeneity with the other tissues. After filtering for samples with available covariates for the differential analyses, we employed 16,151 samples from up to 46 tissues for 932 individuals. GENCODE v26 (73) was used for GTEx as well as the annotation for the proteincoding genes.

\section{Classification of the time of death as day or night and by season}

Using the time of death provided by the GTEx consortium, individuals had been classified between dead during the day or dead during the night if their time of death was falling into the following intervals [08:00-17:00) and [21:00-05:00) respectively (Fig. 1A). Other times of death have been discarded for the day-night analysis to avoid taking into account any RNAseq samples coming from people where the day status was unsure, i.e. twilight. Using this classification, 351, 315 and 266 individuals have been classified as day, night and twilight respectively, of which only 666 individuals (11,527 samples), day and night individuals, were used for differential expression for the day-night cycle. The time corresponding to day and night was manually curated from the Boston (Massachusetts) sunrise and sunset intervals during the year (using https:/www.timeanddate.com/sun/usa/boston). The season of death was provided by the GTEx consortium with 282, 190, 221 and 239 donors that died in fall, spring, summer and winter respectively. Note that for the season, no people were discarded regarding the time of death.

\section{Differential gene expression between day and night}

Differential expression between day and night was performed separately on the 46 tissues using samples from the 666 individuals classified as day or night, ranging from 98 samples (Uterus) to 560 samples (Muscle - Skeletal). Genes were filtered per tissue, removing all genes with a median TPM $<1$ over the day and night samples, leading to 18,022 proteincoding genes expressed in at least one tissue (31,530 genes including all biotypes). The analyses were run using $\mathrm{R}$ v3.6.1 (74), the voom-limma pipeline $(19,75)$ and the TMM 
normalisation method from edgeR $(76,77)$. The significance of the time of death was assessed correcting for the following covariates: sex if the tissue was not a sex-specific tissue; age; body mass index (BMI) and the postmortem interval. All genes with an associate p-value below 0.05 were considered as differentially expressed between night and day. Results, including all biotypes, are available in Supplementary Dataset 1. Even though the number of differentially expressed genes across experiments was affected by the number of samples (Fig. S12A), no effect was observed regarding the ratio of day/night samples and the number of differentially expressed genes between day and night (Fig. S12B).

\section{Differential gene expression between seasons}

Differential expression between seasons was performed separately on the 46 tissues using samples from the 932 individuals, ranging from 139 samples (Brain - Substantia nigra) to 789 samples (Muscle - Skeletal). Genes were filtered and the analyses were run as described in the section 'Differential expression between day and night'. This resulted in a set of 18,018 protein-coding genes expressed in at least one tissue (31,517 genes including all biotypes). The covariates used were the following: time of death classified as: day, night or twilight; sex, if the tissue was not a sex-specific tissue; age; BMI; postmortem interval. The effect of each season was accessed by comparing one season against all the others, leading to four differential expression analyses, one for each season, for the whole dataset. All genes with an associate p-value below 0.05 were considered as seasonal. Results, including all biotypes, are available in Supplementary Dataset 2. Even if the number of seasonal genes was related to the number of samples per the tissue (Fig. S13A), it was not related to the proportion of samples available for a given season over the total. (Fig. S13B).

Comparison of human, baboon and mouse core clock genes

The baboon results were downloaded from Mure et al. (2). We extracted the significant genes using the same threshold as Mure et al. (p-value $\leq 0.05$ ). Common tissues between GTEx and baboon have been manually curated (Table S3). The mouse circadian genes were downloaded from the CirGRDB database (26), which include genes already defined as circadian, and we only selected the genes found by RNA-seq and from the publication with the PubMed ID 25349387, corresponding to the publication by Zhang et al. (1). Common tissues between 
GTEx and the mouse tissues list had been manually curated (Table S4). This dataset was used to compute the number of occurrences of Clock and Npas 2 in mouse tissues.

To perform the comparison of the core clock genes in human vs baboon, we extracted the core clock genes in both species in the 20 common tissues between the GTEx and the baboons' tissue. From this, we analyzed only genes that were significant in both species, i.e. we discarded the genes which were found to be significant in either baboon or human but not in both. The same analysis was done to compare human and mouse.

\section{Functional enrichment analyses}

Protein-coding genes for set enrichment were chosen if they were observed to be seasonal or day-night in at least ten tissues (the direction of the change could differ in different tissues). Then, we used the enrichment analysis tool Enrichr $(78,79)$ and we selected the ten Wikipathway and/or GO functional terms with the highest combined score. The same procedure was used for day-night and seasonal genes. For COVID-19 related gene sets, gene sets with an adjusted $\mathrm{p}$-value greater than 0.05 were discarded.

\section{Circadian and hormone gene sets}

The protein sequences, along with the IDs, of proteins annotated as circadian, either experimentally or by orthology, in human were downloaded from the Circadian Gene DataBase website (http://cgdb.biocuckoo.org/) (28) the 01/26/2021. The correspondence between the Ensembl protein ID and the UniProtKB ID with the Ensembl gene ID was downloaded using BioMart from Ensembl (80). The list of hormone genes was downloaded from Mirabeau et al. (48). The Ensembl peptide IDs were linked to their respective Ensembl gene ID using $\mathrm{R}$ and the biomaRt package $(81,82)$. The gene IDs for hormones with deprecated peptide IDs were retrieved manually using the Ensembl website (http://www.ensembl.org) and the ones that were obscelets were removed. Two hormone genes were added manually: GH1 and LEP. The list is available at Table S11.

\section{Data access}

All GTEx open-access data are available on the GTEx Portal (https://gtexportal.org/home/datasets). All GTEx protected data are available via dbGaP 
(accession phs000424.v8). Access to the raw sequence data is now provided through the AnVIL platform (https://gtexportal.org/home/protectedDataAccess).

\section{Acknowledgments}

We thank the donors and their families for their generous gifts of biospecimens to the GTEx research project; the Genomics Platform at the Broad Institute for data generation. The Genotype-Tissue Expression (GTEx) project was supported by the Common Fund of the Office of the Director of the National Institutes of Health (https://commonfund.nih.gov/GTEx). Manuel Muñoz-Aguirre and Thomas Derrien for their feedback on the manuscript. Diego Garrido Martín for his help on side analysis. Claudia Vivori and Vanessa Vega Méndez for providing help with graphical presentations.

\section{Authors' contributions}

V.W. Major data analysis and visualization, final main and supplementary data and figure production and management, writing of the methods, and revisions. R.S. Design and creation of the study, writing of the main text, major data analysis, questions, interpretations, and visualization of earlier editions. R.A. data visualization and analysis, and helpful discussions. M.I. revisions, intellectual contribution, and final figure production. R.G. revisions, accommodation of the research, and intellectual contribution.

\section{Funding}

The research reported in this publication was supported by the National Human Genome Research Institute of the National Institutes of Health under Awards R01MH101814 and 5U24HG009446, by the Spanish Ministry of Science and Innovation under grants PGC2018094017-B-I00 to R.G. and BFU2017-89201-P to M.I., and by the European Research Council (ERC) under the European Union's Horizon 2020 research and innovation program (ERCStG-LS2-637591 to MI). A.R. is a predoctoral fellow of the CONACYT "Becas al Extranjero" Program of Mexico. We acknowledge support of the Spanish Ministry of Science and Innovation to the EMBL partnership, Centro de Excelencia Severo Ochoa and CERCA Programme / Generalitat de Catalunya.

\section{Disclosure Declaration}


The authors declare no competing financial interests.

\section{Bibliography}

1. R. Zhang, N. F. Lahens, H. I. Ballance, M. E. Hughes, J. B. Hogenesch, A circadian gene expression atlas in mammals: implications for biology and medicine. Proc Natl Acad Sci USA. 111, 16219-16224 (2014).

2. L. S. Mure et al., Diurnal transcriptome atlas of a primate across major neural and peripheral tissues. Science. 359 (2018), doi:10.1126/science.aao0318.

3. M. D. Ruben et al., A database of tissue-specific rhythmically expressed human genes has potential applications in circadian medicine. Sci. Transl. Med. 10 (2018), doi:10.1126/scitranslmed.aat8806.

4. M. S. Ferreira et al., The transcriptional landscape of seasonal coat colour moult in the snowshoe hare. Mol. Ecol. 26, 4173-4185 (2017).

5. K. L. Drew et al., Central nervous system regulation of mammalian hibernation: implications for metabolic suppression and ischemia tolerance. J. Neurochem. 102, 1713-1726 (2007).

6. H. Dardente, D. G. Hazlerigg, F. J. P. Ebling, Thyroid hormone and seasonal rhythmicity. Front Endocrinol (Lausanne). 5, 19 (2014).

7. D. Lomet et al., The impact of thyroid hormone in seasonal breeding has a restricted transcriptional signature. Cell. Mol. Life Sci. 75, 905-919 (2018).

8. E. A. Hanon et al., Ancestral TSH mechanism signals summer in a photoperiodic mammal. Curr. Biol. 18, 1147-1152 (2008).

9. G. Sharon et al., Human Gut Microbiota from Autism Spectrum Disorder Promote Behavioral Symptoms in Mice. Cell. 177, 1600-1618.e17 (2019).

10. Q. Li, Y. Han, A. B. C. Dy, R. J. Hagerman, The gut microbiota and autism spectrum disorders. Front. Cell. Neurosci. 11, 120 (2017).

11. B. Xu et al., Association between winter season and risk of death from cardiovascular diseases: a study in more than half a million inpatients in Beijing, China. BMC Cardiovasc. Disord. 13, 93 (2013).

12. J. P. Pell, S. M. Cobbe, Seasonal variations in coronary heart disease. QJM. 92, 689696 (1999).

13. J. P. Pell, J. Sirel, A. K. Marsden, S. M. Cobbe, Seasonal variations in out of hospital cardiopulmonary arrest. Heart. 82, 680-683 (1999).

14. N. Owens, P. D. McGorry, Seasonality of symptom onset in first-episode schizophrenia. Psychol. Med. 33, 163-167 (2003). 
15. C. G. Watson, T. Kucala, C. Tilleskjor, L. Jacobs, Schizophrenic birth seasonality in relation to the incidence of infectious diseases and temperature extremes. Arch. Gen. Psychiatry. 41, 85-90 (1984).

16. E. F. Torrey, J. Miller, R. Rawlings, R. H. Yolken, Seasonal birth patterns of neurological disorders. Neuroepidemiology. 19, 177-185 (2000).

17. M. Philpot, M. Rottenstein, A. Burns, G. Der, Season of birth in Alzheimer's disease. Br. J. Psychiatry. 155, 662-666 (1989).

18. GTEx Consortium, The GTEx Consortium atlas of genetic regulatory effects across human tissues. Science. 369, 1318-1330 (2020).

19. C. W. Law, Y. Chen, W. Shi, G. K. Smyth, voom: Precision weights unlock linear model analysis tools for RNA-seq read counts. Genome Biol. 15, R29 (2014).

20. C. B. Canto, Y. Onuki, B. Bruinsma, Y. D. van der Werf, C. I. De Zeeuw, The Sleeping Cerebellum. Trends Neurosci. 40, 309-323 (2017).

21. T. A. Bargiello, F. R. Jackson, M. W. Young, Restoration of circadian behavioural rhythms by gene transfer in Drosophila. Nature. 312, 752-754 (1984).

22. L. P. Shearman et al., Interacting molecular loops in the mammalian circadian clock. Science. 288, 1013-1019 (2000).

23. A. C. Liu et al., Redundant function of REV-ERBalpha and beta and non-essential role for Bmall cycling in transcriptional regulation of intracellular circadian rhythms. PLoS Genet. 4, e1000023 (2008).

24. C. Scheiermann, Y. Kunisaki, P. S. Frenette, Circadian control of the immune system. Nat. Rev. Immunol. 13, 190-198 (2013).

25. P.-S. Welz et al., BMAL1-Driven Tissue Clocks Respond Independently to Light to Maintain Homeostasis. Cell. 178, 1029 (2019).

26. X. Li et al., CirGRDB: a database for the genome-wide deciphering circadian genes and regulators. Nucleic Acids Res. 46, D64-D70 (2018).

27. E. L. Bittman, Timing in the testis. J. Biol. Rhythms. 31, 12-36 (2016).

28. S. Li et al., CGDB: a database of circadian genes in eukaryotes. Nucleic Acids Res. 45, D397-D403 (2017).

29. K. M. Klimina et al., The effects of desynchronosis on the gut microbiota composition and physiological parameters of rats. BMC Microbiol. 19, 160 (2019).

30. Y. Xu et al., Long-Range Chromosome Interactions Mediated by Cohesin Shape Circadian Gene Expression. PLoS Genet. 12, e1005992 (2016).

31. M. Preußner et al., Body temperature cycles control rhythmic alternative splicing in mammals. Mol. Cell. 67, 433-446.e4 (2017). 
32. P. R. Jansen et al., Genome-wide analysis of insomnia in 1,331,010 individuals identifies new risk loci and functional pathways. Nat. Genet. 51, 394-403 (2019).

33. T. Penzel, J. W. Kantelhardt, C.-C. Lo, K. Voigt, C. Vogelmeier, Dynamics of heart rate and sleep stages in normals and patients with sleep apnea. Neuropsychopharmacology. 28 Suppl 1, S48-53 (2003).

34. Unable to find information for 811209 .

35. T. Manivasagam et al., Role of oxidative stress and antioxidants in autism. Adv. Neurobiol. 24, 193-206 (2020).

36. Y. Martínez et al., The role of methionine on metabolism, oxidative stress, and diseases. Amino Acids. 49, 2091-2098 (2017).

37. J.-H. Ko, C. Prassinos, D. Keathley, K.-H. Han, Novel aspects of transcriptional regulation in the winter survival and maintenance mechanism of poplar. Tree Physiol. 31, 208-225 (2011).

38. E. Dorcey et al., Context-dependent dual role of SKI8 homologs in mRNA synthesis and turnover. PLoS Genet. 8, e1002652 (2012).

39. K. Tenney et al., Drosophila Rtf1 functions in histone methylation, gene expression, and Notch signaling. Proc Natl Acad Sci USA. 103, 11970-11974 (2006).

40. A. Alpsoy, E. C. Dykhuizen, Glioma tumor suppressor candidate region gene 1 (GLTSCR1) and its paralog GLTSCR1-like form SWI/SNF chromatin remodeling subcomplexes. J. Biol. Chem. 293, 3892-3903 (2018).

41. L. Liu et al., A whole genome screen for HIV restriction factors. Retrovirology. 8, 94 (2011).

42. D. N. Slenter et al., WikiPathways: a multifaceted pathway database bridging metabolomics to other omics research. Nucleic Acids Res. 46, D661-D667 (2018).

43. W. Roth et al., Keratin 1 maintains skin integrity and participates in an inflammatory network in skin through interleukin-18. J. Cell Sci. 125, 5269-5279 (2012).

44. K. A. McGowan, S. Aradhya, H. Fuchs, M. H. de Angelis, G. S. Barsh, A mouse keratin 1 mutation causes dark skin and epidermolytic hyperkeratosis. J. Invest. Dermatol. 126, 1013-1016 (2006).

45. M. Zimova et al., Function and underlying mechanisms of seasonal colour moulting in mammals and birds: what keeps them changing in a warming world? Biol. Rev. Camb. Philos. Soc. 93, 1478-1498 (2018).

46. A. Tendler et al., Hormone seasonality in medical records suggests circannual endocrine circuits. Proc Natl Acad Sci USA. 118 (2021), doi:10.1073/pnas.2003926118.

47. H. E. Watts, Seasonal regulation of behaviour: what role do hormone receptors play? Proc. Biol. Sci. 287, 20200722 (2020). 
48. O. Mirabeau et al., Identification of novel peptide hormones in the human proteome by hidden Markov model screening. Genome Res. 17, 320-327 (2007).

49. R. Bao et al., Genome sequencing and transcriptome analyses of the Siberian hamster hypothalamus identify mechanisms for seasonal energy balance. Proc Natl Acad Sci USA. 116, 13116-13121 (2019).

50. D. Vandael, N. V. Gounko, Corticotropin releasing factor-binding protein (CRF-BP) as a potential new therapeutic target in Alzheimer's disease and stress disorders. Transl. Psychiatry. 9, 272 (2019).

51. M. J. Cullen, N. Ling, A. C. Foster, M. A. Pelleymounter, Urocortin, corticotropin releasing factor-2 receptors and energy balance. Endocrinology. 142, 992-999 (2001).

52. J. H. Jhamandas, V. Goncharuk, Role of neuropeptide FF in central cardiovascular and neuroendocrine regulation. Front Endocrinol (Lausanne). 4, 8 (2013).

53. C. Geven, M. Kox, P. Pickkers, Adrenomedullin and Adrenomedullin-Targeted Therapy As Treatment Strategies Relevant for Sepsis. Front. Immunol. 9, 292 (2018).

54. P. J. Hart, E. L. Squires, K. J. Imel, T. M. Nett, Seasonal variation in hypothalamic content of gonadotropin-releasing hormone $(\mathrm{GnRH})$, pituitary receptors for $\mathrm{GnRH}$, and pituitary content of luteinizing hormone and follicle-stimulating hormone in the mare. Biol. Reprod. 30, 1055-1062 (1984).

55. C. M. Schooling, J. C. M. Ng, Reproduction and longevity: A Mendelian randomization study of gonadotropin-releasing hormone and ischemic heart disease. SSM Popul. Health. 8, 100411 (2019).

56. S. Bhatia, S. Bhatia, J. Mears, G. Dibu, A. Deshmukh, Seasonal periodicity of ischemic heart disease and heart failure. Heart Fail. Clin. 13, 681-689 (2017).

57. A. W. Fischer, B. Cannon, J. Nedergaard, Leptin: is it thermogenic? Endocr. Rev. 41 (2020), doi:10.1210/endrev/bnz016.

58. R. K. Srivastava, A. Krishna, Adiposity associated rise in leptin impairs ovarian activity during winter dormancy in Vespertilionid bat, Scotophilus heathi. Reproduction. 133, 165-176 (2007).

59. S. Cahill, E. Tuplin, M. R. Holahan, Circannual changes in stress and feeding hormones and their effect on food-seeking behaviors. Front. Neurosci. 7, 140 (2013).

60. J. Lázaro, M. Hertel, C. C. Sherwood, M. Muturi, D. K. N. Dechmann, Profound seasonal changes in brain size and architecture in the common shrew. Brain Struct. Funct. 223, 2823-2840 (2018).

61. J. C. Walton, A. Haim, J. M. Spieldenner, R. J. Nelson, Photoperiod alters fear responses and basolateral amygdala neuronal spine density in white-footed mice (Peromyscus leucopus). Behav. Brain Res. 233, 345-350 (2012).

62. M. A. Hofman, D. F. Swaab, Seasonal changes in the suprachiasmatic nucleus of man. 
Neurosci. Lett. 139, 257-260 (1992).

63. A. T. McKenzie et al., Brain Cell Type Specific Gene Expression and Co-expression Network Architectures. Sci. Rep. 8, 8868 (2018).

64. P. G. Ferreira et al., The effects of death and post-mortem cold ischemia on human tissue transcriptomes. Nat. Commun. 9, 490 (2018).

65. C. Nosal, A. Ehlers, J. A. Haspel, Why lungs keep time: circadian rhythms and lung immunity. Annu. Rev. Physiol. 82, 391-412 (2020).

66. H. Kang, T. M. Kenealy, R. E. Cohen, The hypothalamic-pituitary-gonadal axis and thyroid hormone regulation interact to influence seasonal breeding in green anole lizards (Anolis carolinensis). Gen. Comp. Endocrinol. 292, 113446 (2020).

67. F. J. P. Ebling, On the value of seasonal mammals for identifying mechanisms underlying the control of food intake and body weight. Horm. Behav. 66, 56-65 (2014).

68. J. C. Walton, Z. M. Weil, R. J. Nelson, Influence of photoperiod on hormones, behavior, and immune function. Front. Neuroendocrinol. 32, 303-319 (2011).

69. S. Kumar, T. J. Cunningham, G. Duester, Nuclear receptor corepressors Ncor1 and Ncor2 (Smrt) are required for retinoic acid-dependent repression of Fgf8 during somitogenesis. Dev. Biol. 418, 204-215 (2016).

70. V. Perissi, K. Jepsen, C. K. Glass, M. G. Rosenfeld, Deconstructing repression: evolving models of co-repressor action. Nat. Rev. Genet. 11, 109-123 (2010).

71. J. R. Joseph-Vanderpool et al., Abnormal pituitary-adrenal responses to corticotropinreleasing hormone in patients with seasonal affective disorder: clinical and pathophysiological implications. J. Clin. Endocrinol. Metab. 72, 1382-1387 (1991).

72. A. S. P. Lim et al., Diurnal and seasonal molecular rhythms in human neocortex and their relation to Alzheimer's disease. Nat. Commun. 8, 14931 (2017).

73. J. Harrow et al., GENCODE: the reference human genome annotation for The ENCODE Project. Genome Res. 22, 1760-1774 (2012).

74. R. C. Team, R: A Language and Environment for Statistical Computing (2019).

75. M. E. Ritchie et al., limma powers differential expression analyses for RNAsequencing and microarray studies. Nucleic Acids Res. 43, e47 (2015).

76. D. J. McCarthy, Y. Chen, G. K. Smyth, Differential expression analysis of multifactor RNA-Seq experiments with respect to biological variation. Nucleic Acids Res. 40, 4288-4297 (2012).

77. M. D. Robinson, D. J. McCarthy, G. K. Smyth, edgeR: a Bioconductor package for differential expression analysis of digital gene expression data. Bioinformatics. 26, 139-140 (2010).

78. E. Y. Chen et al., Enrichr: interactive and collaborative HTML5 gene list enrichment 
analysis tool. BMC Bioinformatics. 14, 128 (2013).

79. M. V. Kuleshov et al., Enrichr: a comprehensive gene set enrichment analysis web server 2016 update. Nucleic Acids Res. 44, W90-7 (2016).

80. D. Smedley et al., The BioMart community portal: an innovative alternative to large, centralized data repositories. Nucleic Acids Res. 43, W589-98 (2015).

81. S. Durinck et al., BioMart and Bioconductor: a powerful link between biological databases and microarray data analysis. Bioinformatics. 21, 3439-3440 (2005).

82. S. Durinck, P. T. Spellman, E. Birney, W. Huber, Mapping identifiers for the integration of genomic datasets with the R/Bioconductor package biomaRt. Nat. Protoc. 4, 1184-1191 (2009).

83. A. Lachmann et al., Geneshot: search engine for ranking genes from arbitrary text queries. Nucleic Acids Res. 47, W571-W577 (2019).

84. M. V. Kuleshov et al., The COVID-19 Drug and Gene Set Library. Patterns (N Y). 1, 100090 (2020). 


\section{Main Figures}
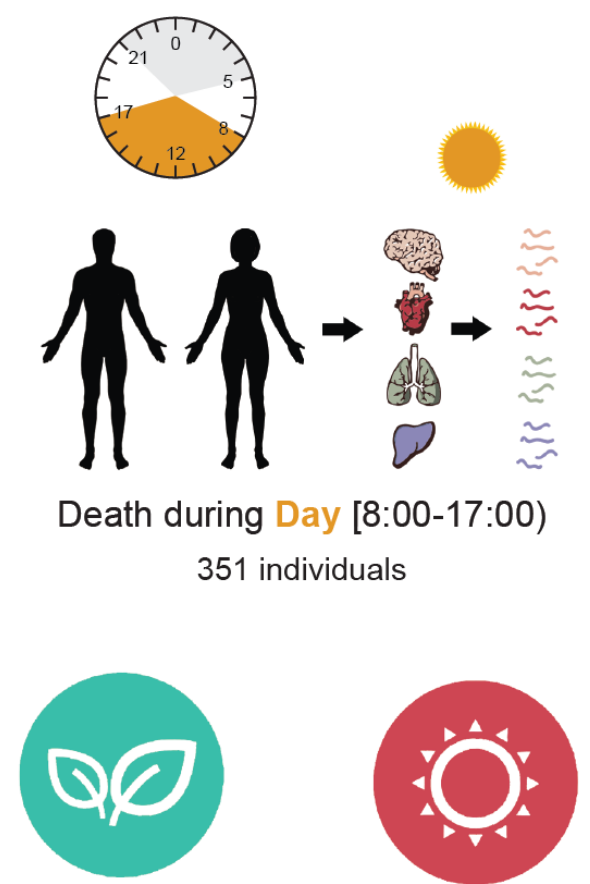

Spring

190 individuals

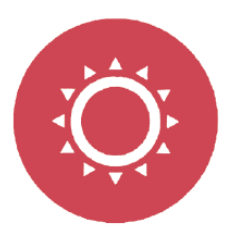

Summer

221 individuals
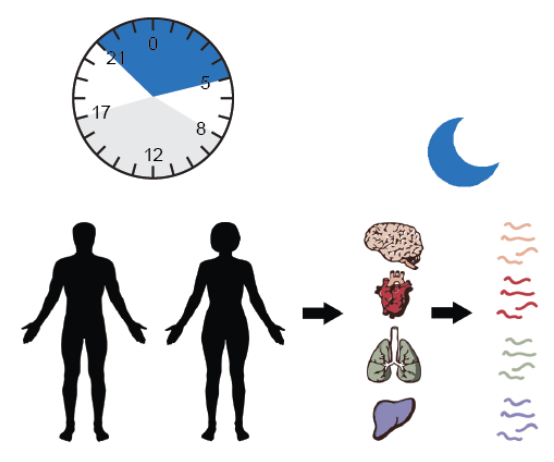

Death during Night [21:00-5:00)

315 individuals

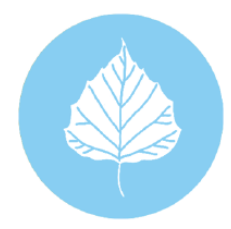

Fall

282 individuals

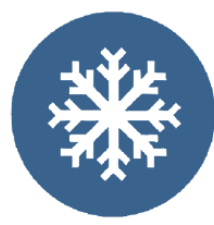

Winter

239 individuals

Fig. 1: Day-night and seasonal classification of the GTEx samples. (A) Samples from GTEx were classified as either day or night depending on the time of death of the donor, respectively between 08:00 and 17:00 (351 individuals) and between 21:00 and 05:00 (315 individuals). (B) Samples from GTEx were assigned to the different seasons (spring, summer, fall and winter), according to the reported season of death of the donor. 


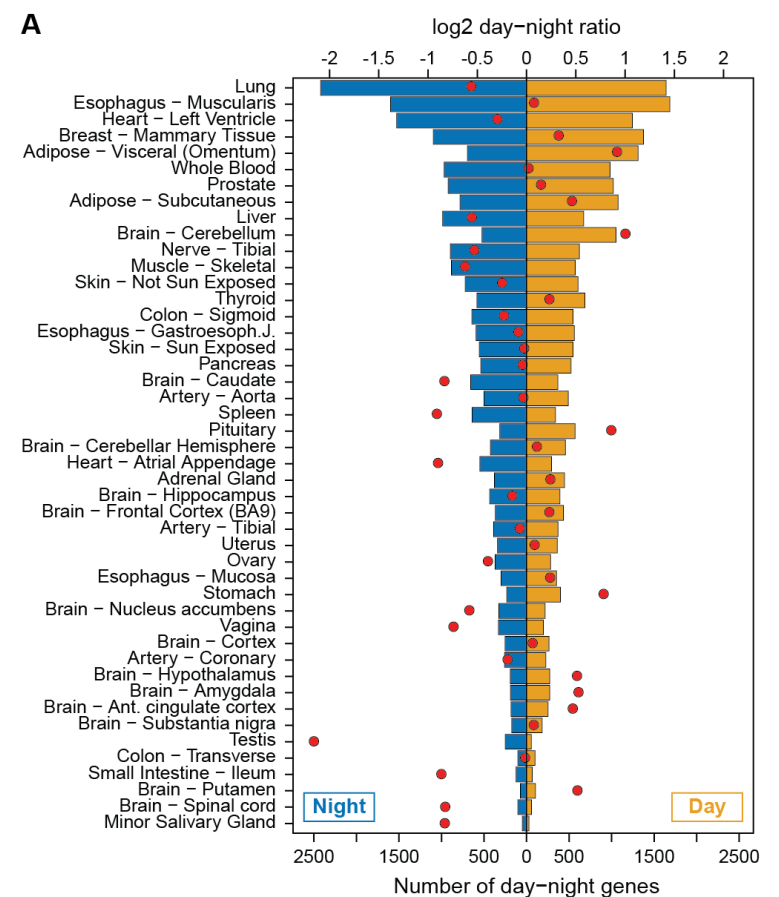

B

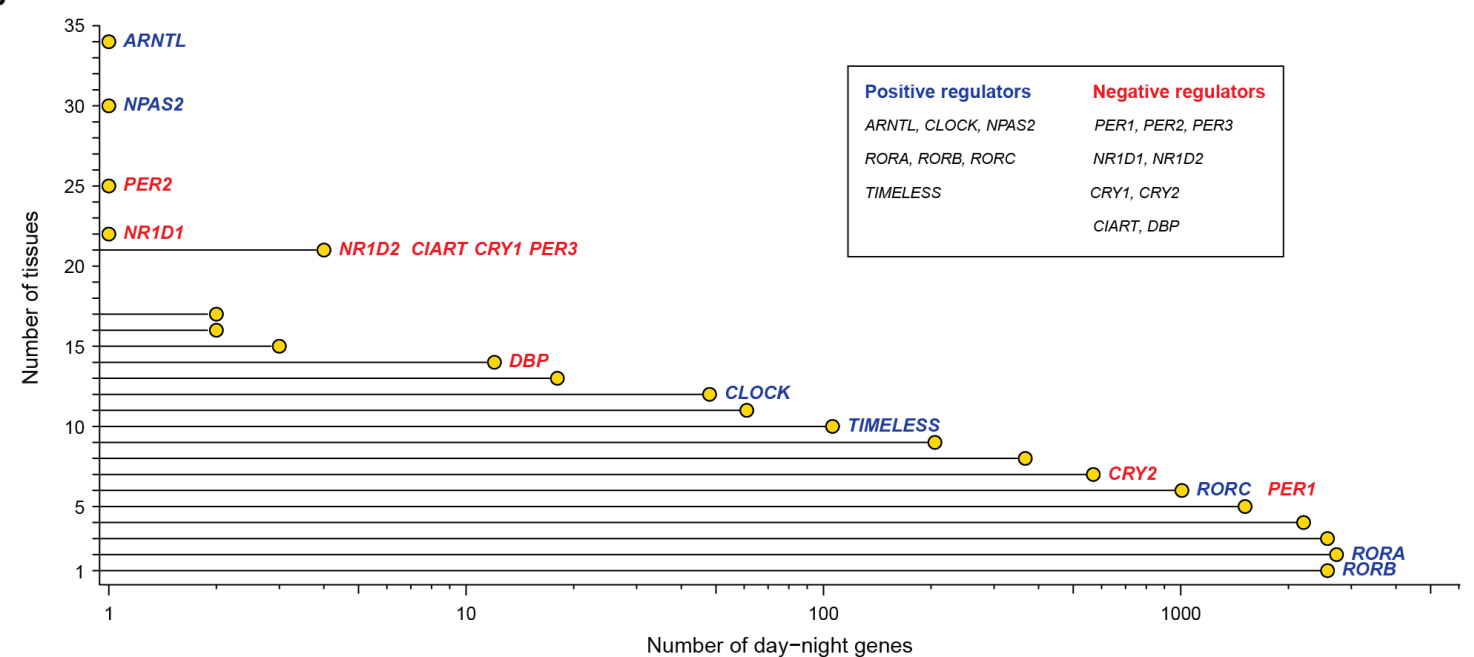

C

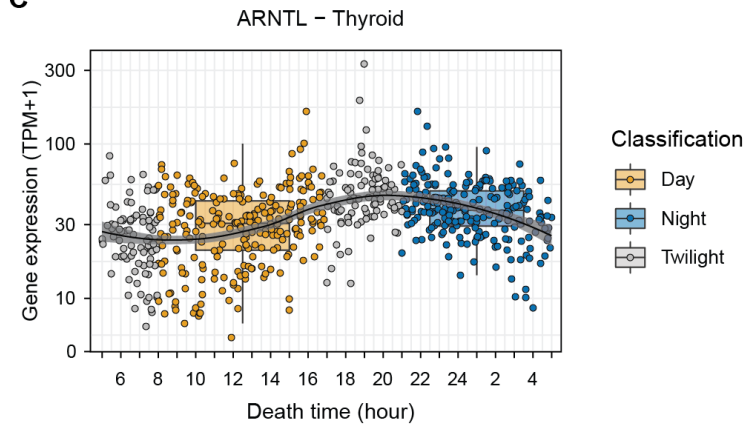

D

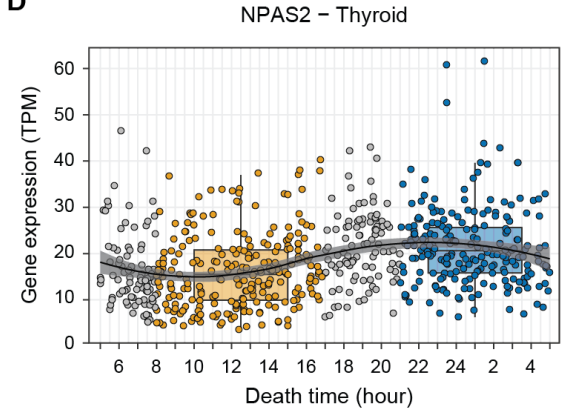

Classification

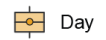

Eight

E. Twilight

Fig. 2: Distribution of the day-night genes in the GTEx tissues. (A) Number of genes found as day-night, i.e. genes differentially expressed between day and night (barplots; bottom $\mathrm{x}$-axis), and $\log 2$ ratio between the number of genes up- and down-regulated at day time (red dots; top x-axis) for each tissue. Tissues are sorted by the total number of day-night genes. (B) Distribution of the number of tissues (y-axis) in which genes are classified as day-night (xaxis, $\log _{10}$ scale). Core clock genes are highlighted. (C) Expression of $A R N T L$ in the thyroid in GTEx samples at the time of death of the GTEx donors (in hours). The colors of the dots represent the classification of the individuals according to their time of death: during the day 

made available under aCC-BY-NC 4.0 International license.

(yellow), during the night (blue), or in-between for twilight (grey). The samples classified as twilight were discarded for the day-night analysis. The "circadian" curve was created using the geom_smooth function from ggplot2 in R with the 'loess' method. 
bioRxiv preprint doi: https://doi.org/10.1101/2021.02.28.433266; this version posted March 1, 2021. The copyright holder for this preprint (which was not certified by peer review) is the author/funder, who has granted bioRxiv a license to display the preprint in perpetuity. It is made available under aCC-BY-NC 4.0 International license.

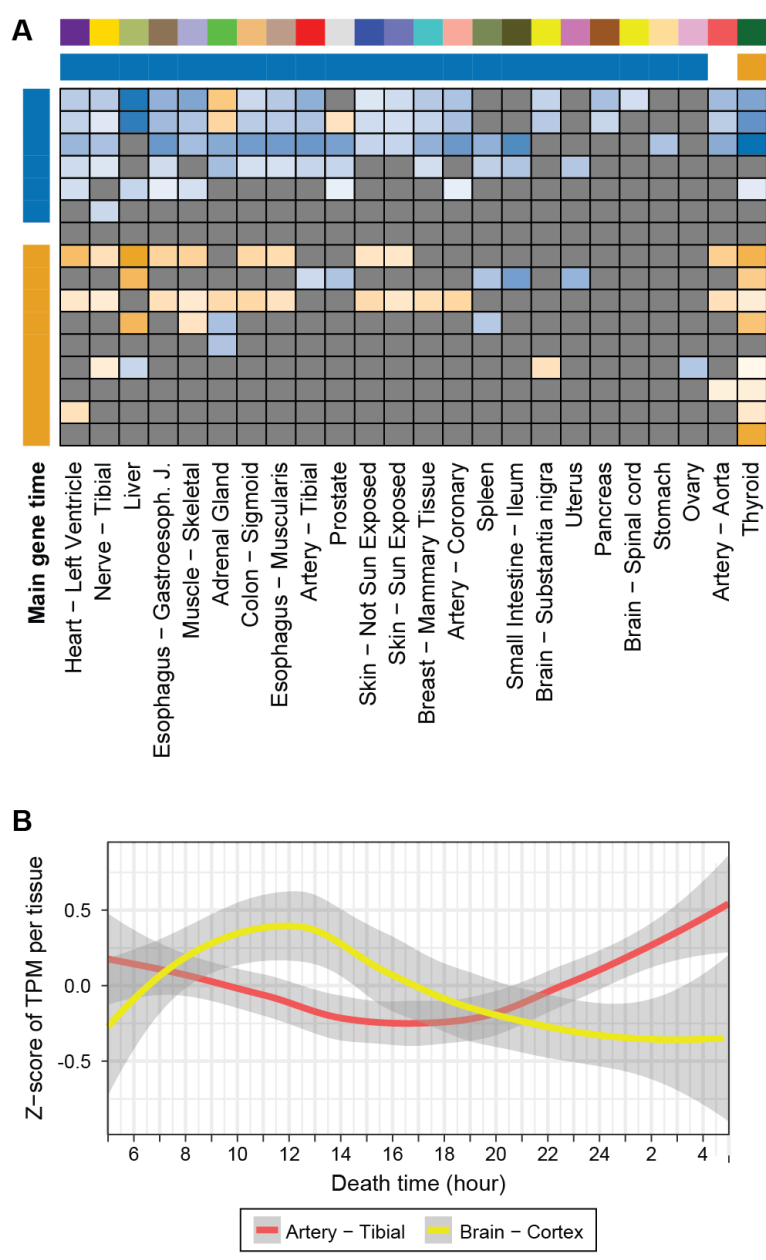

C
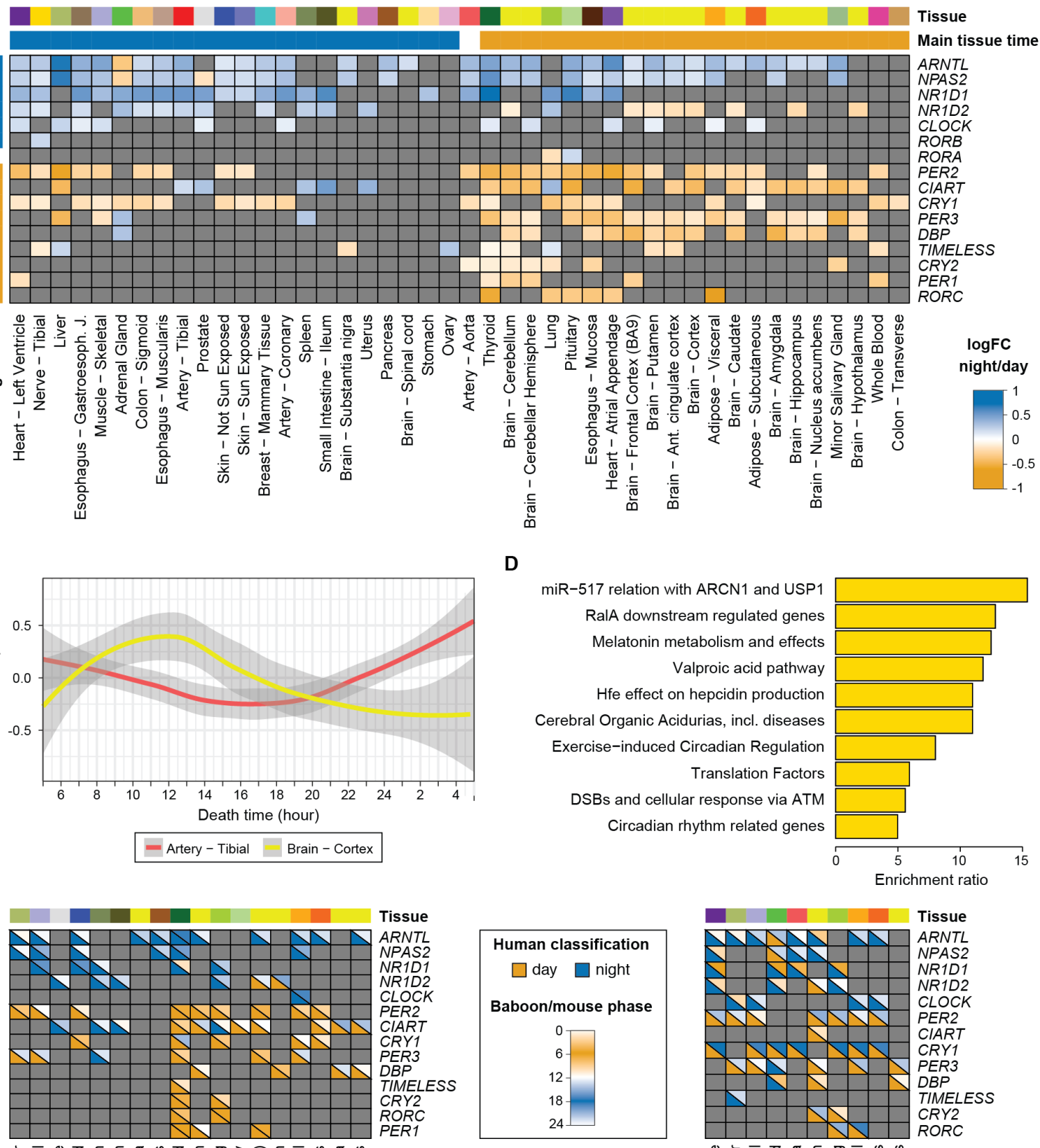

D
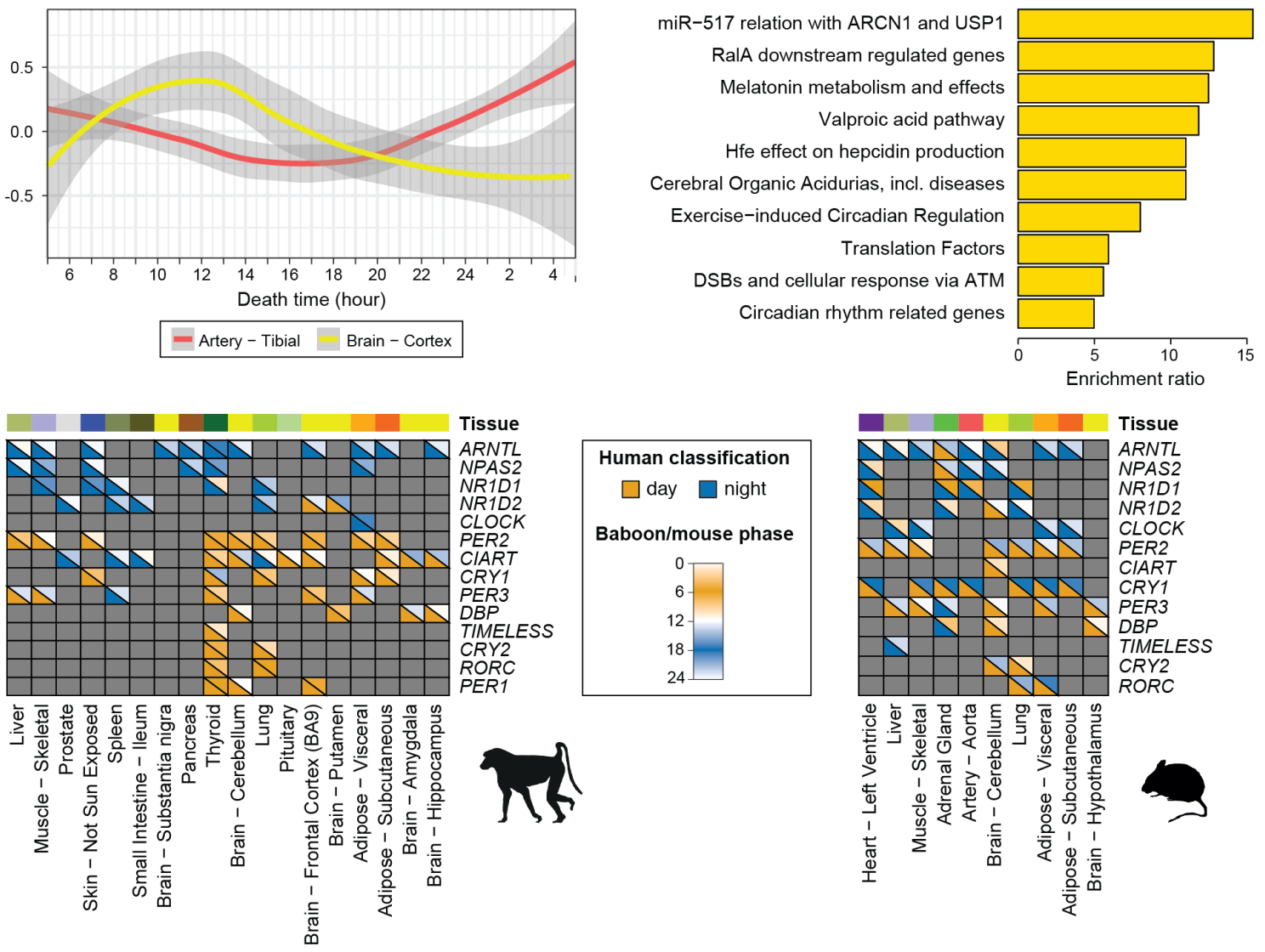

Fig. 3: Day/night differential expression of the core clock genes in human, baboon, and

mouse. (A) Day/night differential expression of the 16 core clock genes in GTEx tissues. Cells in the matrix are colored according to the log fold-change obtained with the voom-limma pipeline, from yellow (day) to blue (night). Genes without significant effects were colored in 
grey. In addition, we labelled each gene ("Main gene time") as day (night) if it was upregulated in more tissues during the day (night) than during the night (day). We labelled the tissues similarly ("Main tissue time") depending on the number of genes that were upregulated during the day (night) on that tissue. One gene, RORA, and one tissue, the aorta, were upregulated in the same number of times during the day and the night, and have not been labelled. Genes and tissues have been sorted according to i) their main time and ii) their number of significant effects. (B) Curves obtained from the Z-score of the TPM using the geom smooth function from ggplot2 in R with the 'loess' method for NR1D2 in the artery tibial (red) and in the brain - cortex (yellow). (C) Comparison of human and baboon (left) and human and mouse (right) orthologous core clock genes in common tissues. Only gene/tissue pairs that are significant for both compared species are shown. Cells are separated into two with i) on the bottom left, the day-night classification of the gene in the tissue in human, and ii) on the top right, the baboon/mouse phase obtained from Mure et al. (2) or Li et al. (26), respectively. (D) Enriched Wikipathway functions for day-night genes present in at least ten tissues. The 10 terms from Wikipathways with the highest combined score from Enrichr are shown. 

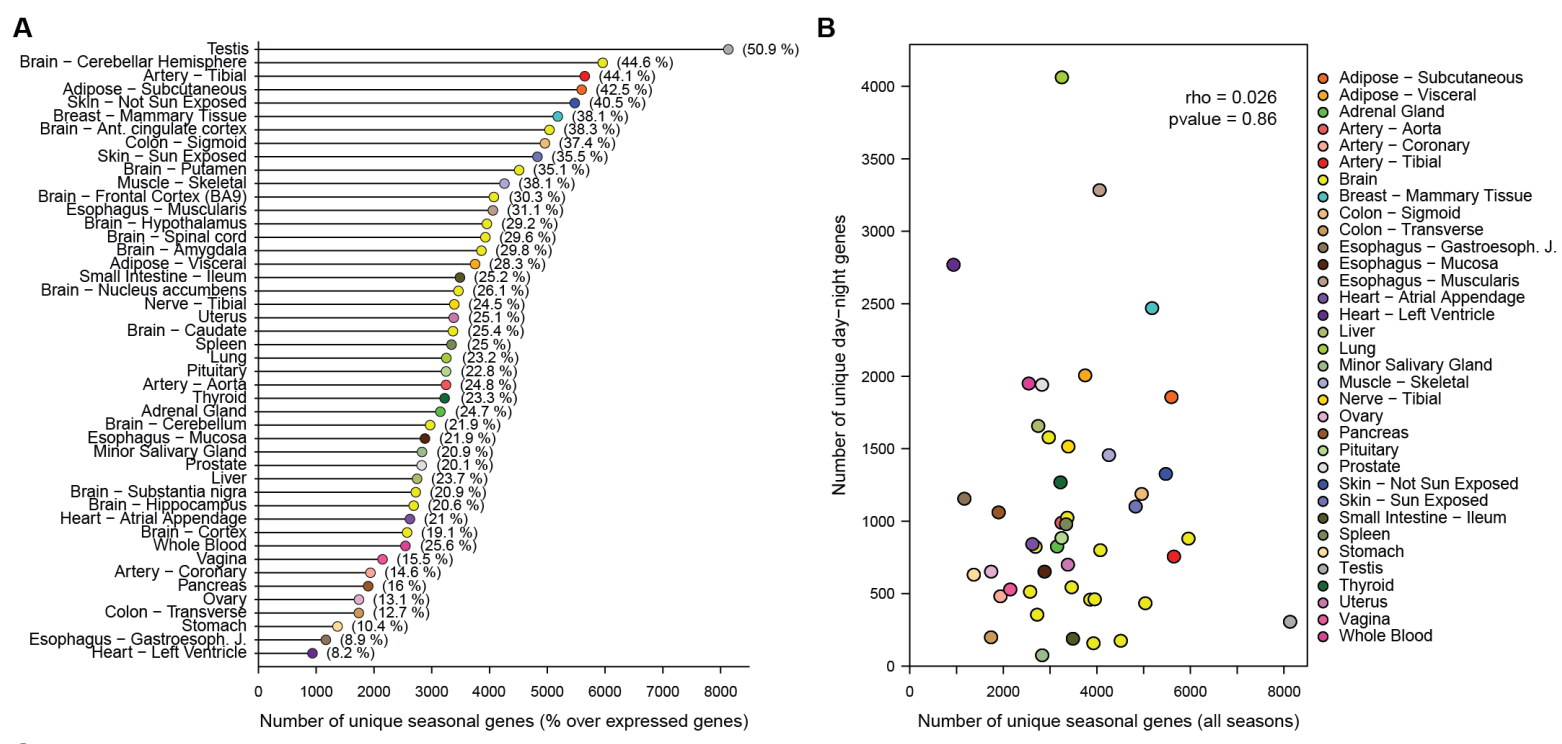

C
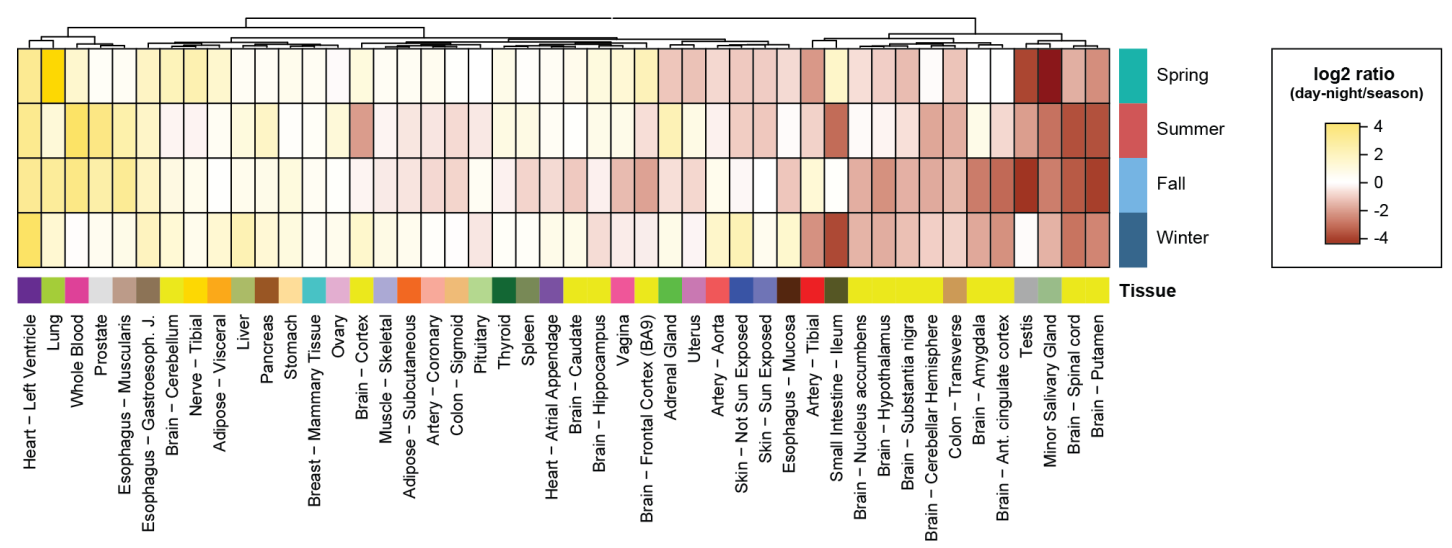

Fig. 4: Distribution of the seasonal genes in the GTEx tissues. (A) Number of unique genes found as seasonal (x-axis), i.e. genes differentially expressed in at least one season when compared to the others, per tissue (y-axis). The numbers in parentheses represent the percentage of unique seasonal genes in a given tissue over the number of expressed genes in that tissue. (B) Number of unique seasonal vs. day-night genes per tissue. Statistics from a Spearman's correlation are shown. (C) $\log 2$ ratio between the number of day-night genes over seasonal genes for each tissue and season separately. The higher the $\log 2$ value, the more daynight genes compare to the number of seasonal genes. Tissues were clustered using Euclidean distance and the Ward's method. 
bioRxiv preprint doi: https://doi.org/10.1101/2021.02.28.433266; this version posted March 1, 2021. The copyright holder for this preprint (which was not certified by peer review) is the author/funder, who has granted bioRxiv a license to display the preprint in perpetuity. It is made available under aCC-BY-NC 4.0 International license.

A

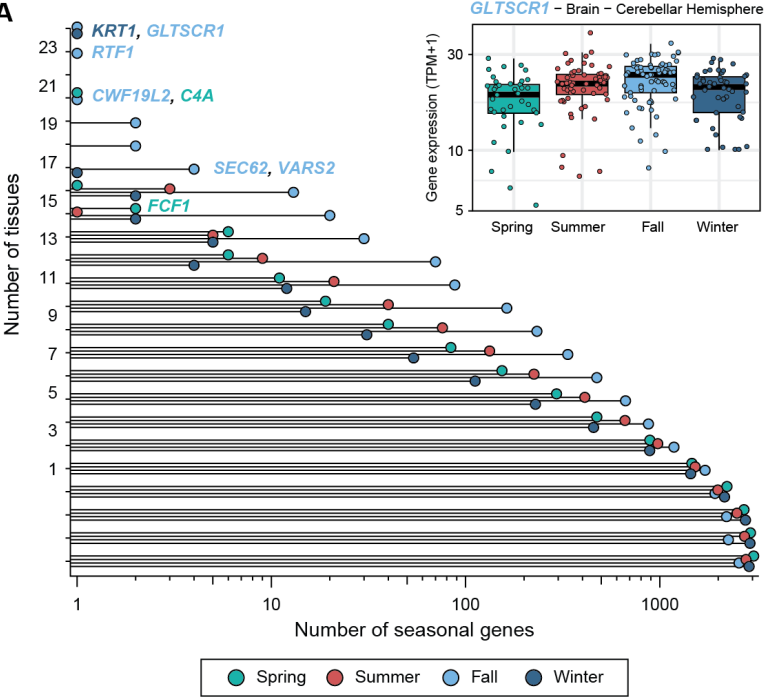

C

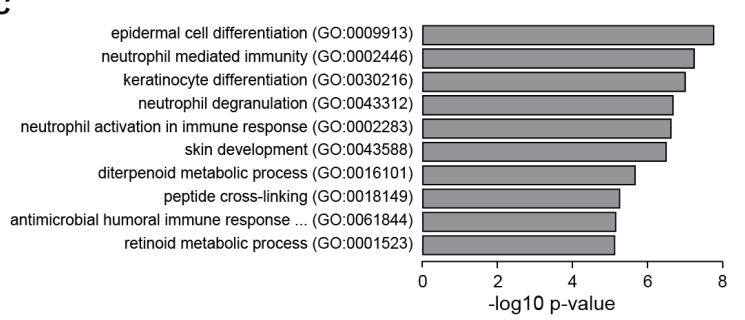

D

B

Vitamin B6-dep. and responsive disorders Molybdenum cofactor (Moco) biosynnthesis
Methionine De Novo and Salvage Pathway Sulindac Metabolic athway Eukaryotic Transcription Inititition Irinotecan Pathway
Vitamin B12 Disorders Copper homeostasis Electron Transport Chain SRF and miRs in Smooth Muscle Cell Differentiation - Index Ctoplasmic Ribosomal Proteins Trans-sulfuration pathway Met metabolism to Sulphur Amino Acids ranscription and translation at the HIV-1 LTR

Mitochondral complex l assembly model Endometrial cancer mRNA Processing

EV release from cardiac cells

Dual hijack model of Vif in HIV infection Ethanol metabolism producing ROS by CYP2E1 Hypertrophy Model Iron metabolism in placenta

Photodynamic therapy-induced UPR Apoptosis-related network in ovarian cancer Corticotropin-releasing hormone signaling pathway Parkin-Ubiquitin Proteasomal System pathway
VEGFA-VEGFR2 Signaling Pathway
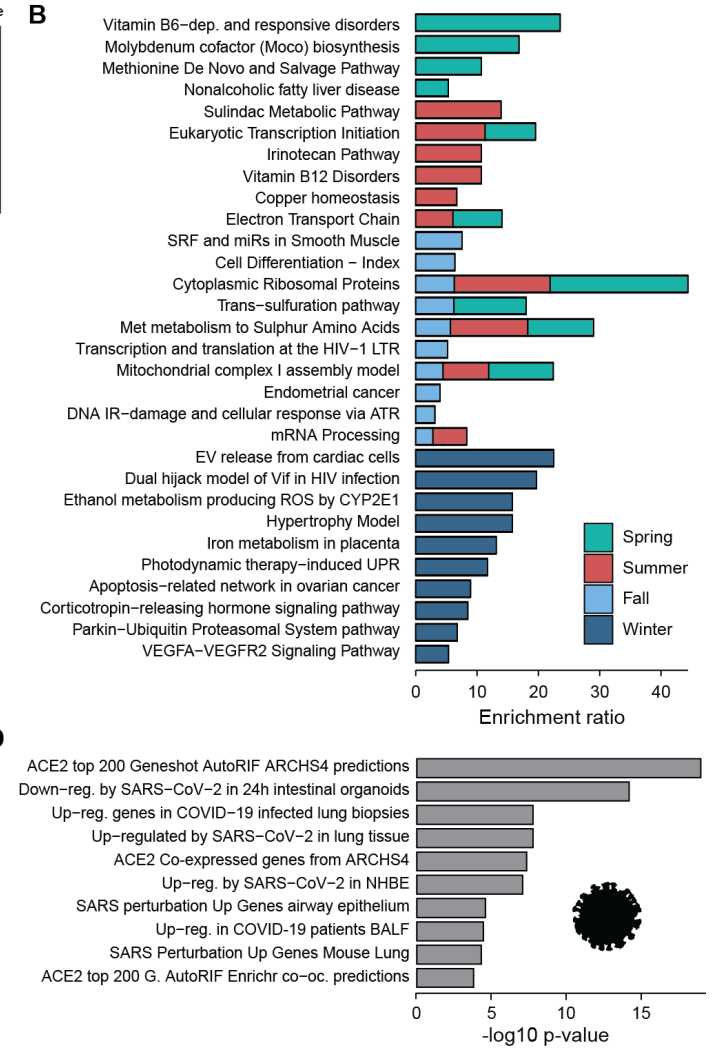

E
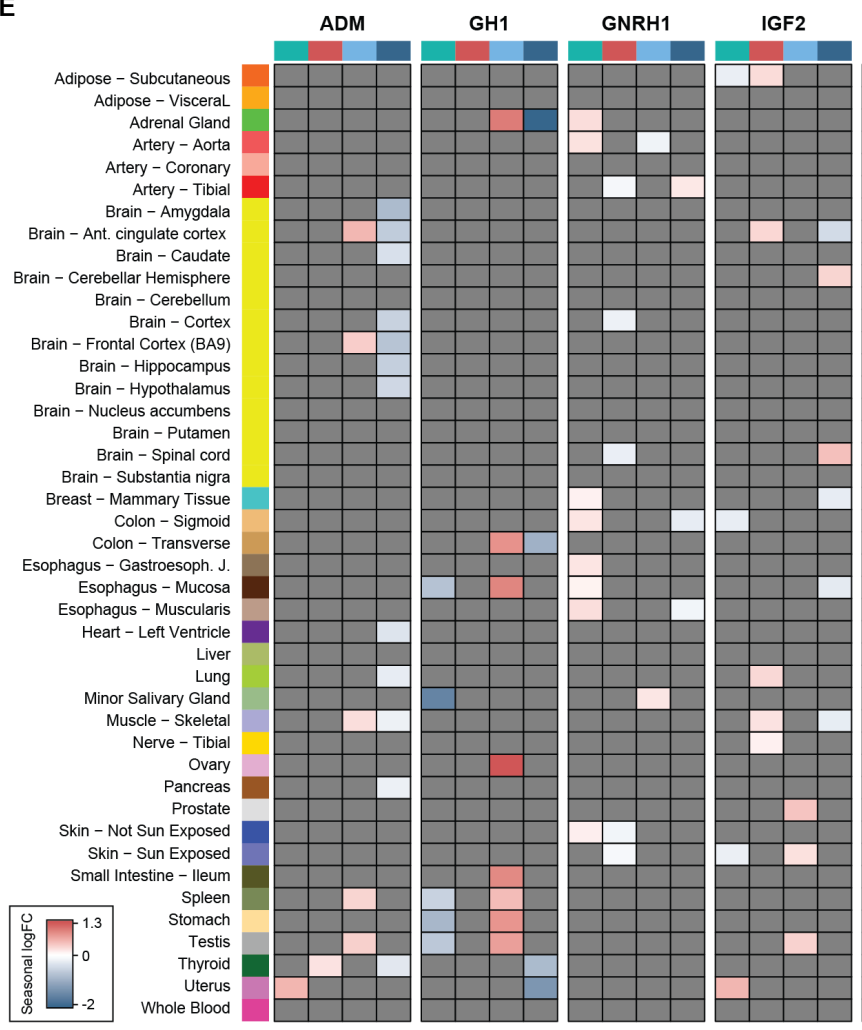

NMB
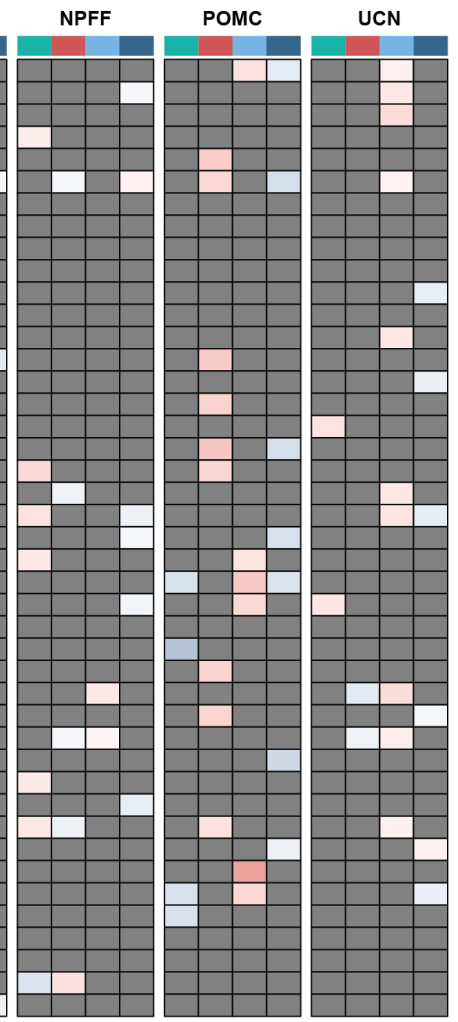

Fig. 5: Seasonal genes and associated functions. (A) Distribution of the number of tissues (x-axis, $\log _{10}$ scale) in which genes were classified as seasonal (red: summer, green: spring, 
light blue: fall, dark blue: winter). The names of the genes found, for a given season, in at least 20 tissues (KRT1, GLTSCR1, RTF1, C4A, CWF19L2, SEC62,VARS2) or in at least 15 non-brain tissues $(K R T 1, C 4 A, F C F 1)$ are shown and their color correspond to their respective season. (B) Enriched Wikipathway functions for seasonal genes (red: summer, green: spring, light blue: fall, dark blue: winter) present in at least ten tissues. The ten terms from Wikipathways with the highest combined score from Enrichr for each season separately are shown. (C) Gene Ontology enrichment of Biological Processes of the set of 192 strongly seasonal genes as computed by Enrichr $(78,79)$. (D) Enrichment in COVID-19 related genes of the 192 strongly seasonal genes as computed by Enrichr. ACE2 Geneshot AutoRIF ARCHS4 predictions were obtained by Geneshot (83) combining genes previously published to be associated with $A C E 2$, as well as genes predicted to be associated with ACE2 based on data integration from multiple sources, including co-expressoin matrices based on RNAseq data and others (84). (E) Seasonal log fold-change of the eight hormone-coding genes that show seasonal effect in at least 14 season/tissue pairs. Genes without significant effects are colored in grey. 

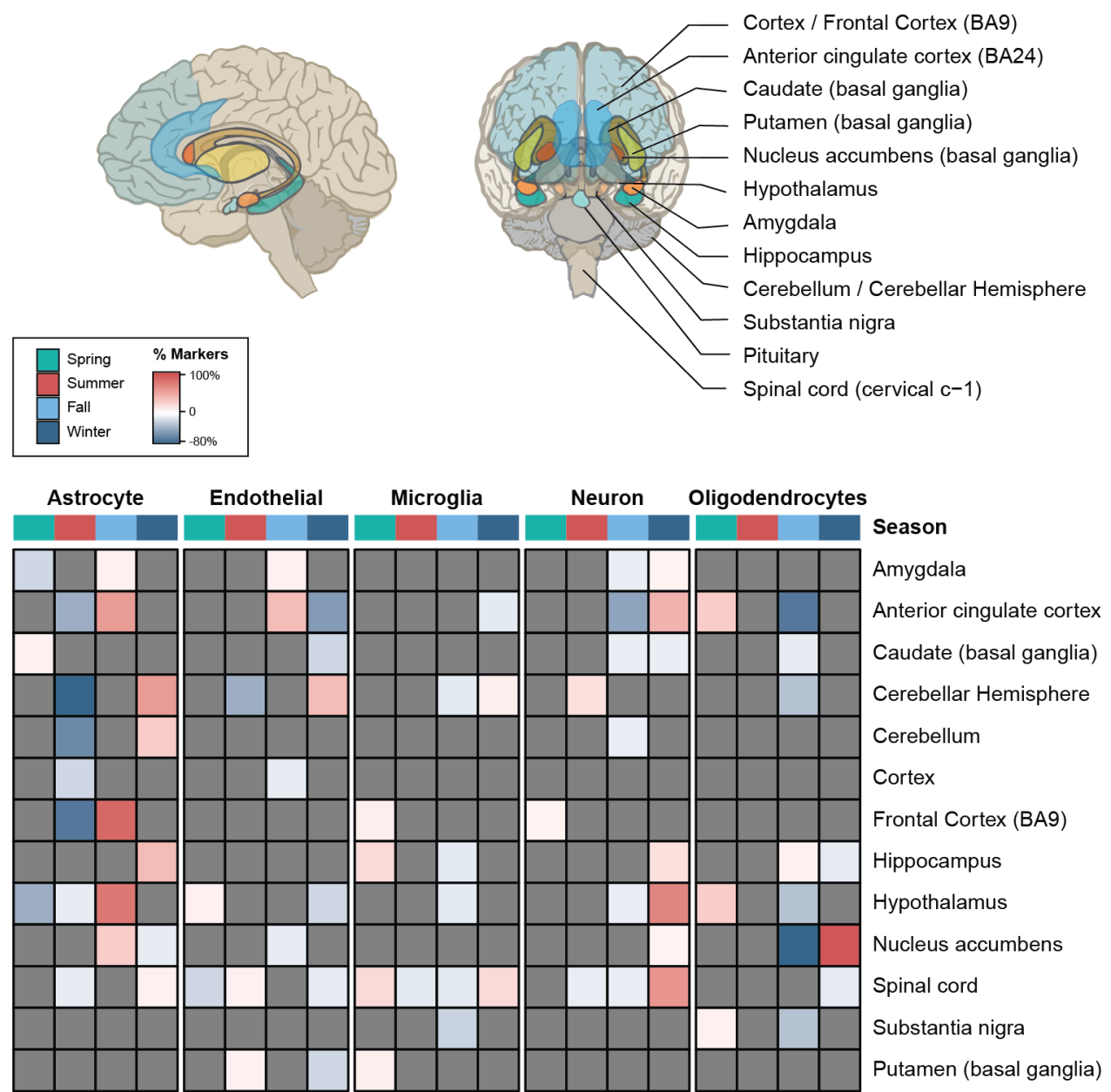

Fig. 6: Seasonal variation in cell-type specific markers across human brain regions. Percentage of the marker genes under-expressed (blue) or over-expressed (red) for five brain cell types: astrocyte, endothelial, microglia, neuron, and oligodendrocytes, in brain subregions (depicted in the brain scheme above, downloaded and modifed from the GTEx web portal). All significant markers for a cell type for a specific season in a subregion were differentially expressed in the same direction, over or under expressed. Markers without significant effects were colored in grey. 